Curr Treat Options Gastroenterol. 2019 March ; 17(1): 48-62. doi:10.1007/s11938-019-00216-7.

\title{
New Developments in the Diagnosis and Treatment of Eosinophilic Esophagitis
}

\author{
Quan M. Nhu, M.D., Ph.D ${ }^{1,2,{ }^{*}}$ and Fouad J. Moawad, M.D ${ }^{1, *}$ \\ ${ }^{1}$ Division of Gastroenterology \& Hepatology, Scripps Clinic, La Jolla, CA, USA \\ ${ }^{2}$ Scripps Research Translational Institute, The Scripps Research Institute, La Jolla, CA, USA
}

\section{Abstract}

Purpose of Review: Eosinophilic esophagitis (EoE) is a chronic, allergen-driven, immunemediated disease of the esophagus that progresses to esophageal fibrostenosis if left untreated. The aim of this review is to provide a concise update on recent clinically relevant advances in the development of diagnostic and therapeutic approaches for EoE.

Recent Findings-Current diagnostic and disease monitoring protocols for EoE rely on repetitive endoscopic evaluations and esophageal tissue acquisition for histopathologic analysis. Recent advancements in EoE diagnosis include endoscopic functional lumen imaging probe (FLIP), transnasal endoscopy (TNE), and the emergence of non-invasive diagnostic tools including cytosponge, esophageal string test, and mucosal impedance probe. Biomarkers for EoE have not yet proven their clinical utility. No Food and Drug Administration (FDA)-approved drugs currently exist for the treatment of EoE. Topical corticosteroid, proton-pump inhibitors (PPI), elimination diet, and dilation are the current treatment modalities for confirmed EoE. Promising results from clinical trials are emerging for biologic agents that target the interleukin (IL)-13 and the IL-4/IL-13 receptor, specifically, RPC4046 and dupilumab, respectively.

Summary-New diagnostic algorithms, non-invasive diagnostic strategies, and treatment modalities for EoE are emerging. Patients with EoE continue to require a multimodal and multidisciplinary management approach.

\section{Keywords}

Eosinophilic esophagitis (EoE); Diagnosis; Treatment; Corticosteroids; elimination diet; biologic therapy

\section{INTRODUCTION}

Eosinophilic esophagitis (EoE) is a chronic, allergen-driven, immune-mediated disease of the esophagus $[1,2]$. There is a male predominance, occurring at a male-to-female ratio of

\footnotetext{
*Corresponding authors: Quan M. Nhu, MD, PhD, Division of Gastroenterology \& Hepatology, Scripps Clinic, 10666 N. Torrey Pines Road, Suite 203N, La Jolla, CA 92037 Tel.: 858-554-8880, Nhu.Quan@ scrippshealth.org, Fouad J. Moawad, M.D. Division of Gastroenterology, Scripps Clinic, 10666 N. Torrey Pines Road, Suite 203N, La Jolla, CA 92037 Tel.: 858-824-4151,

Moawad.Fouad@scrippshealth.org.

Conflict of Interest: None
} 
3:1. The incidence of EoE has been increasing [3, 4]. Persistent, sub-optimally controlled esophageal inflammation underlies a natural progression to fibrostenotic disease; during which esophageal dysmotility, tissue rigidity, reduced tissue compliance, and stricture formation often dictate the clinical presentations of dysphagia and food impactions [5-9].

EoE creates a significant healthcare burden and an impaired quality of life [10-14]. Children with EoE can exhibit failure to thrive with feeding difficulty, abdominal pain, food regurgitation, and, on rare occasions, esophageal food impaction [7, 15, 16]. Dysphagia and food impactions, however, are often the presenting symptoms in adult EoE patients [17]. The combination of abnormal feeding behaviors, feeding difficulty, food aversion, recurrent dysphagia, and food impaction likely contribute to failure to thrive in children and also to the lower body mass index in adults $[18,19]$. However, children with uncomplicated EoE appear to have normal nutritional intake despite feeding behavior dysfunction [20].

Despite improvement in the understanding and recognition of EoE, there remains a significant delay to diagnosis from the time of symptom onset [21]. Such diagnostic delay is detrimental to the patient, since the risk of esophageal stricturing disease increases with each year of delayed diagnosis [7]. Nevertheless, care must be exercised to diagnose EoE accurately, as having the diagnosis can be life transforming and potentially raise life insurance premiums [22-24]. The aim of this review is to provide a concise update on recent advances in the development of diagnostic and therapeutic approaches of EoE that have contributed to our rapidly evolving understanding of EoE.

\section{Diagnosis of EoE}

EoE is currently defined as the presence of both clinical symptoms of esophageal dysfunction and histologic evidence of significant esophageal eosinophilic inflammation with 15 or more eosinophils per high-power field (hpf), although other infiltrating immune cells such as mast cells, lymphocytes, basophils, and innate lymphoid cells likely also contribute to disease pathogenesis $[9,25,26]$. The inflammation must be isolated to the esophagus and other causes of esophageal eosinophilia need to be excluded [26].

Emerging visual diagnostic tools-Direct visualization of the esophageal lumen and mucosa by upper endoscopy not only provides useful diagnostic information, but also provides the physician with the ability to immediately obtain esophageal biopsies for tissue diagnosis and disease stratification, i.e., inflammatory stage, fibrostenotic disease, or mixed disease states. The EoE Endoscopic Reference Score (EREFS) classification is a validated tool to standardize quantification and reporting of endoscopic findings of disease severity: edema, rings, exudates, furrows, and strictures [27-29]. Since EoE is a patchy disease, 2 to 4 biopsies each in the distal and proximal halves or, occasionally the distal, middle, and proximal thirds, of the esophagus are typically obtained and put in separate biopsy jars [30, 26]. One emerging caveat of esophageal tissue acquisition is that esophageal biopsies often lack adequate lamina propria where subepithelial fibrosis occurs and thus may underestimate the severity of EoE, i.e., pathologic remodeling and fibrostenotic disease, at the time of tissue acquisition for diagnosis. A recent analysis showed that adequate lamina propria was present in only $42 \%$ of esophageal biopsies [31]. Therefore, at least 7 biopsy specimens 
from the middle-distal esophageal segment have been recently suggested to optimally detect EoE subepithelial fibrosis [31].

Transnasal endoscopy (TNE) is a recent diagnostic tool that may potentially become widely adapted by clinicians given its safety, cost effectiveness, feasibility, and high patient preference [32]. TNE is performed in an unsedated patient, and while using only topical anesthetics, TNE is able to provide histologic tissue of the proximal esophagus. In the study performed in pediatric patients with EoE, the total surface area from mucosal biopsies was not significantly different when compared with the same subjects undergoing upper endoscopy using standard endoscopy forceps [32]. More than half the patients and the majority of the parents preferred this approach over standard endoscopy with sedation. Further validation in larger cohorts is needed.

Upper gastrointestinal (GI) esophagram double-contrast protocol, which includes swallowing a $12.5-\mathrm{mm}$ barium tablet, was recently proposed as a complementary study to upper endoscopy to detect fibrostenotic changes in EoE [33]. However, the need for specialized radiographic interpretation capability may restrict its use to only a few select centers.

Endoscopic functional lumen imaging probe (FLIP) is a novel and widely accepted endoscopic method to assess esophageal caliber and distensibility in EoE patients. FLIP uses high-resolution impedance planimetry during volume-controlled distention to determine variations in luminal pressure and geometry in a cross-sectional area of the esophagus along an axial plane $[34,35]$. FLIP studies in EoE patients have demonstrated reduced esophageal distensibility [8], which is associated with increased food impactions and the need for esophageal dilation [36-38]. Esophageal narrowing and reduced esophageal distensibility are features also observed in pediatric EoE [8], suggesting that early diagnosis of EoE and timely treatment to prevent pathologic tissue remodeling are required. FLIP has established its importance in EoE severity assessment, disease stratification, and assessment of treatment response.

Emerging non-invasive diagnostic tools-Due to the invasive nature of repetitive endoscopic evaluations with multiple biopsies, minimally invasive methods to diagnose EoE and to assess disease activity have been proposed. Cytosponge, a string-tethered spherical mesh sponge that is compressed in a dissolvable gelatin capsule, is swallowed by an unsedated patient and retrieved by withdrawing the string through the mouth [39]. The cytosponge is safe, well tolerated, and the esophageal tissue specimen obtained by this method appears adequate for histopathologic analysis. The sensitivity and specificity of the cytosponge to assess EoE histologic activity are $75 \%$ and $86 \%$, respectively [39, 40]. Similarly, the esophageal string test captures adherent luminal secretions containing eosinophil-derived proteins that reflect mucosal inflammation in EoE [41]. Other approaches similar to the cytosponge and esophageal string test methods, such as endoscopic esophageal brushings and blind esophageal brushings via a nasogastric tube, have been recently proposed $[42,43]$. Extending the analysis of esophageal brushings to include esophageal levels of eosinophil-derived neurotoxin (EDN), which is highly expressed in the EoE esophagus [44], appears to improve EoE detection and disease monitoring [43]. Further 
investigation, optimization and validation of these emerging non-invasive diagnostic tools in larger patient cohort remain to be established.

Measurement of electrical impedance at the esophageal mucosal surface is another emerging modality to assess disease activity in EoE [45-48]. The mucosal impedance probe detects changes in the electrical impedance, thought to be related to a defect in the esophageal barrier function. Real-time mucosal impedance measurements correlate inversely with esophageal eosinophil counts and spongiosis severity in EoE, allowing the ability to quickly determine and monitor EoE disease activity [49]. However, upper endoscopy is still required to introduce the mucosal impedance probe into the esophagus.

Potential biomarkers-Measurement of serum levels of select panels of EoE-related cytokines, chemokines, and serum proteins has not yet proven their clinical utility as serologic biomarkers for EoE [50-52]. However, EoE patients have increased mean peripheral blood absolute eosinophil counts (AEC) [53-55]. Normalized serum eosinophil peroxidase levels, the ratio of serum eosinophil peroxidase levels to AEC, are lower in EoE patients and have an inverse correlation with esophageal eosinophil density; and thus, are being evaluated as a potential biomarker for EoE [53]. The presence of multiple foodspecific serum IgE antibodies correlates with esophageal eosinophilia in children with nonspecific gastrointestinal symptoms, which can be useful in stratifying potential EoE patients [56]. Esophageal and plasma food antigen- specific IgG4 antibodies are elevated in EoE [57-60]. Furthermore, serum epithelial-specific autoantibodies, specifically, antidesmoglein 3 (DSG3) IgG4 and anti-collagen XVII (NC16A) IgG4, are elevated in EoE. Serum anti-NC16A IgG4, but not IgG1, levels are significantly elevated in EoE patients and decrease after topical corticosteroid treatment in histologic responders [61]. While promising, additional investigation and validation are required.

\section{Treatment of EoE}

There are currently no drugs approved by the Food and Drug Administration (FDA) for the treatment of EoE. The complexity of current EoE management requires a multimodal, multidisciplinary approach that includes chronic corticosteroid treatment, proton-pump inhibitors (PPI), dietary antigen restriction, lifestyle modification, and repeated endoscopic diagnostic and therapeutic evaluations [25, 62]. Several biologic agents are being investigated for the management of EoE. A paradigm-shifting advancement is the recent orphan designation of dupilumab, a monoclonal antibody that targets the a-chain of interleukin (IL)-13 and IL-4 receptor [63]. Prior to dupilumab's orphan status for EoE, efforts in the management of EoE have focused on: 1) controlling inflammation and tissue remodeling with corticosteroids, proton-pump inhibitors (PPI), and various investigative biologic agents; 2) the exclusion of antigenic stimulation via elimination diet; and 3) endoscopic dilation of the symptomatic, fibrostenotic esophagus if uncontrolled by medical and dietary therapies [64]. Data have emerged to suggest that in adult EoE patients, controlling esophageal inflammation may decrease the need for subsequent esophageal dilation $[38,65]$.

Topical Corticosteroids-Controlling inflammation with swallowed topical corticosteroids is a mainstay of EoE treatment [25]. Emerging proprietary and non- 
proprietary corticosteroid formulations that improve bioavailability and treatment efficacy should increase available treatment options [66-68]. In the United States, fluticasone administered as an aerosolized and swallowed formulation and oral viscous preparations of budesonide are the two most frequently used topical corticosteroids for EoE [69, 70]. Clinical trials in Europe have demonstrated successful use of an effervescent budesonide tablet in EoE [68]. In a recent study, oral dispersible budesonide induced histologic remission in $87 \%$ at 12 weeks in adults with EoE [71]. Topical corticosteroid dampens EoEassociated esophageal inflammation, improves mucosal barrier integrity and histologic remodeling, resulting in improvement of esophageal diameter, distensibility, and in the rates of food impaction [72-75, 38]. Oral viscous budesonide therapy achieves better endoscopic and histologic improvement, compared to fluticasone propionate in pediatric and adolescent EoE; this is likely due to oral viscous corticosteroid preparations achieving better esophageal mucosal deposition [76, 66]. Oral viscous budesonide (OVB) can also be compounded by specialty pharmacy. In a retrospective analysis of a cohort of 48 children and adult EoE patients who received compounded OVB at $2.4 \mathrm{mg}$ mean initial dose (range $1-6 \mathrm{mg}$ per day) either once daily or twice daily, there was a significant, durable symptomatic, endoscopic, and histologic response after a mean follow-up period of 17 months [77]. In children with EoE, long-term maintenance dosing of aerosolized and swallowed fluticasone was found to be safe and achieved improvement in histologic inflammation, lamina propria fibrosis, endoscopic features and clinical symptoms [75].

Recently, an induction-maintenance corticosteroid protocol was proposed for EoE management [78]. In this protocol for adult EoE patients, induction corticosteroid therapy with $1 \mathrm{mg}$ twice daily of budesonide is initiated for 2 to 4 weeks to reach clinical response, followed by maintenance therapy with $0.25 \mathrm{mg}$ twice daily that can then be rescued with 1 $\mathrm{mg}$ twice daily dosing for 7 to 15 days during EoE flares. Corticosteroids are discontinued if patient maintains $\ 6$ months of deep remission - a combination of clinical symptom improvement; endoscopic inflammatory remission with complete absence of white exudates, furrows, and edema; and histological inflammatory remission with peak eosinophil count less than 5 eosinophils per high-power field. Using this protocol, deep remission was achieved at 89 weeks in $9.4 \%$ of adult EoE patients. Corticosteroid discontinuation occurred at 104.7 weeks, with $81.8 \%$ experiencing EoE clinical relapse after a median of 22.4 weeks. In another study, budesonide maintenance dosing at once every other day in adult EoE patients was not effective [79]. These studies suggest that a higher daily budesonide maintenance dose is necessary to potentially achieve complete histologic and endoscopic remission.

Asymptomatic esophageal candidiasis and herpes esophagitis have been observed with swallowed, topical corticosteroid therapy [80,75]. In clinical trials, asymptomatic esophageal candidiasis occurred in approximately $20 \%(0 \%$ - 26\%) of topical corticosteroidtreated EoE patients [81]. Adrenal insufficiency of unclear clinical significance has been reported in children on high-dose swallowed topical fluticasone propionate or oral viscous budesonide $[82,83]$.

Proton-pump inhibitor (PPI) therapy for EoE-High-dose PPI therapy is no longer a diagnostic agent, but is now widely accepted as a first-line therapeutic modality for EoE, 
based on our evolving understanding of PPI-responsive esophageal eosinophilia (PPI-REE) being in the EoE continuum [84-88, 26]. Treatment with PPI modulates esophageal inflammation beyond suppression of the acidic environment [89, 90]. A recent meta-analysis of 33 studies, comprising 619 patients with symptomatic esophageal eosinophilia, demonstrated that PPI therapy induced clinical response and histologic remission in $60.8 \%$ and $50.5 \%$ of patients, respectively [91]. On PPI maintenance, sustained remission has been observed in 73-86\% of pediatric and adult PPI-REE patients [92]. Low-dose PPI maintenance with esomeprazole at $1 \mathrm{mg} / \mathrm{kg}$ (maximum of $40 \mathrm{mg}$ ) daily dosing in $57 \mathrm{EoE}$ children, who responded initially to an 8-week esomeprazole trial at $1 \mathrm{mg} / \mathrm{kg}$ dose (maximum of $40 \mathrm{mg}$ ) twice daily, achieved clinical response in $86 \%$ and histologic remission in $70.1 \%$ of these children at 1 year with an adequate safety profile [93]. Complete histologic response, defined as $\mathbf{5}$ eosinophils/hpf, to the 8-week PPI induction phase resulted in better histologic control on the low-dose PPI maintenance. Histologic remission was sustained at 2 years in 11 of 12 children (91.6\%) from the same cohort who underwent further esomeprazole maintenance dose reduction to $0.5 \mathrm{mg} / \mathrm{kg}$ once daily in the second 12-month period. Although PPI adverse effects, i.e., diarrhea, abdominal pain, headache, and urticaria, are mild and infrequent [93], high-dose induction therapy is likely required to achieve therapeutic benefits of inflammation modulation and mucosal barrier restoration; thus, tapering the maintenance dose to the lowest effective dose should remain a treatment goal to minimize adverse drug outcomes.

Elimination diets in EoE-Dietary modification to exclude food-derived antigenic stimulation is effective in achieving histologic and clinical remission in EoE patients [9496]. Elemental diet remains the most effective strategy; however, therapy compliance is a significant challenge. Four weeks of elemental diet improves clinical symptoms in $88 \%$ and achieves histologic response in $71 \%$ of patients, with an improvement in mucosal barrier integrity and a significant reduction in gene expression of key cytokines such as IL-13, IL-5, and thymic stromal lymphopoietin (TSLP) [97, 98]. Esophageal inflammation is improved in $88 \%$ of EoE patients by elemental diet therapy; in $74 \%$ by six-food-elimination diet (SFED) excluding cow milk, wheat, egg, soy, peanut and seafood; and in $64 \%$ by a fourfood elimination diet (FFED) strategy excluding only milk, wheat, egg, and soy [99, 100]. Antigenic exclusion can also improve esophageal distensibility [38]. A meta-analysis of 33 studies on 1128 children and 189 adult EoE patients shows that elemental diets are effective for $90.8 \%$ of cases, SFED for $72.1 \%$, and allergy testing-directed elimination diet for $45.5 \%$ of cases [96]. However, allergy testing-directed selective elimination diet by way of skin prick tests and atopy patch testing has been shown to have suboptimal efficacy in adult EoE and thus is generally not recommended [101, 102]. More recently, esophageal prick test (EPT) with food antigens was proposed a potential novel safe and feasible method to guide elimination diets [103], however, EPT remains to be further evaluated and validated.

While effective, empiric food elimination by way of elemental diet, SFED, or FFED may cause unnecessary dietary restriction. A novel step-up food elimination strategy was recently proposed. In a multicenter, prospective clinical trial, a 2-4-6 step-up elimination diet strategy in EoE patients achieved clinicohistologic response and identified food triggers of EoE early, and thus avoided unnecessary dietary restriction [104]. Briefly, patients were 
initiated on a 2-food elimination diet (TFED) excluding milk and gluten-containing cereals first. Non-responders were stepped up sequentially to FFED, with the additional exclusion of egg and legumes; and ultimately to SFED, with the elimination of nuts and fish/seafood. Each elimination diet step lasted 6 weeks. Overall, EoE remission was achieved in $43 \%$ of the TFED group, in $60 \%$ of patients receiving both TFED and FFED, and in $79 \%$ of patients receiving all three tiers of elimination diet therapy. Patients who failed any elimination diet therapy were rescued with 8 weeks of swallowed topical steroids - viscous oral budesonide $2 \mathrm{mg}$ twice daily in adults and $1 \mathrm{mg}$ twice daily in children, or swallowed fluticasone $800 \mu \mathrm{g}$ twice daily. Histologic remission occurred in $79 \%$ of patients who underwent corticosteroid rescue therapy following a failed step-up antigen elimination protocol [104]. This strategy reduced the number of endoscopies when compared to a traditional six-food elimination diet. However, declaring elimination diet failure after 6-week trial was recently suggested to be premature, with histologic resolution eventually observed when the elimination diet therapy was extended for an additional 5 to 22 weeks or a mean of 10 weeks [105]. The duration of elimination diet, goal-directed therapy will likely need to be revisited in a larger study.

Relevant biologic therapies-Targeting TNF-a with infliximab or IgE with omalizumab has not been shown to be effective in treating EoE [25]. An anti-IL-13 monoclonal antibody, QAX576, reduced esophageal eosinophilia and EoE-related gene expression, but did not significantly improve clinical symptoms [25]. Another anti-IL- 13 monoclonal antibody, RPC4046, however, reduced esophageal eosinophilia, but also improved endoscopic features and dysphagia in EoE patients, particularly in steroid-refractory EoE patients [25]. Dupilumab, an antibody that targets the common a-chain of the IL-13 and IL-4 receptor, recently achieved an orphan designation for the treatment of EoE. In a 12-week phase II, randomized, double-blind, placebo-controlled clinical trial in 47 adult patients with active, moderate-to-severe EoE, dupilumab at $600 \mathrm{mg}$ subcutaneous loading dose, followed by 300 mg subcutaneous injection once weekly, achieved clinical response with a 3-point reduction (45\% improvement) in Straumann Dysphagia Instrument score at week 10 of dupilumab versus 1.3-point reduction (19\% improvement) for placebo; histologic remission with overall peak intraepithelial eosinophil count reduced by $93 \%$ from baseline for dupilumab versus an increase in $14 \%$ from baseline for placebo; and endoscopic and esophageal distensibility improvement [63].

Dilation Therapy for EoE-The narrowed, fibrostenotic, symptomatic EoE-associated esophagus can be rescued with dilation in a slow, deliberate, and graduate manner to improve dysphagia [106, 107]. In a recent study of $509 \mathrm{EoE}$ patients, esophageal dilation was well tolerated [108]. Mucosal tear is expected and is a dilation success rather than a complication $[106,109]$. Traditionally a procedure usually performed in adult EoE patients, dilation in EoE children was shown recently to be safe, with pain being a commonly reported post-procedure complaint [110]. Overall, the risk of esophageal perforation due to dilation is less than $1 \%$ [109]. In a meta-analysis of 845 children and adults with EoE undergoing 1820 dilations, the pooled clinic response was $95 \%$ with a very low complication rate (perforation in $0.38 \%$, hemorrhage in $0.05 \%$, and hospitalization in 
$0.67 \%$ ) [111]. The predictor of repeated, multiple dilations was a smaller baseline esophageal diameter, with the second dilation typically occurring within 1 year [108].

\section{CONCLUSION}

Recent advances in EoE are improving our diagnostic and therapeutic approaches. Current diagnostic strategy still relies on endoscopic evaluation with esophageal biopsies for tissue acquisition and histopathologic analysis. Although endoscopic biopsies potentially require deeper tissue penetration to include the lamina propria to more accurately characterize EoE severity, up-and-coming investigative diagnostic approaches are shifting toward non-invasive modalities. Current treatment strategies available to EoE patients center on monotherapy or combination therapy, utilizing dietary modification to exclude antigenic stimulation and corticosteroids and PPI to control tissue inflammation and pathologic tissue remodeling. Dilation therapy for the narrowed, fibrostenotic, symptomatic esophagus can potentially be avoided with optimal medical and elimination diet therapies, although may be required for severe fibrostenotic strictures as an adjunct therapy. Dupilumab is an emerging promising treatment for EoE that has gained orphan drug status. Given the complexity of the treatment regimens and frequent follow ups, optimal care of EoE patients requires a multimodal, multi-disciplinary management approach.

\section{ACKNOWLEDGEMENTS}

QMN is supported by Scripps Research Translational Institute NIH/NCATS CTSA Award 5 UL1 TR001114, 5KL2 TR001112, and by a CEGIR award. CEGIR (Consortium of Eosinophilic Gastrointestinal Disease Researchers; U54 AI1 17804) is part of the Rare Disease Clinical Research Network (RDCRN), an initiative of the Office of Rare Diseases Research (ORDR), NCATS, and is funded through collaboration between NIAID, NIDDK, and NCATS. CEGIR is also supported by patient advocacy groups including APFED, CURED, and EFC. QMN and FJM are supported by Scripps Clinic and by Scripps Clinic Medical Group Research \& Education Awards.

Funding: QMN is supported by Scripps Research Translational Institute NIH/NCATS CTSA Award 5 UL1 TR001114, 5KL2 TR001112, and by a CEGIR award. CEGIR (Consortium of Eosinophilic Gastrointestinal Disease Researchers; U54 AI117804) is part of the Rare Disease Clinical Research Network (RDCRN), an initiative of the Office of Rare Diseases Research (ORDR), NCATS, and is funded through collaboration between NIAID, NIDDK, and NCATS. CEGIR is also supported by patient advocacy groups including APFED, CURED, and EFC. QMN and FJM are supported by Scripps Clinic and by Scripps Clinic Medical Group Research \& Education Awards.

\section{Abbreviations:}

$\begin{array}{ll}\text { AEC } & \text { absolute eosinophil count } \\ \text { EoE } & \text { eosinophilic esophagitis } \\ \text { FLIP } & \text { functional lumen imaging probe } \\ \text { FFED } & \text { four-food elimination diet } \\ \text { hpf } & \text { high-power field } \\ \text { PPI } & \text { proton-pump inhibitor } \\ \text { PPI- REE } & \text { PPI-responsive esophageal eosinophilia } \\ \text { SFED } & \text { six-food elimination diet }\end{array}$


TFED two-food elimination diet

TNE transnasal endoscopy

\section{REFERENCES}

1. Dellon ES, Hirano I. Epidemiology and Natural History of Eosinophilic Esophagitis. Gastroenterology 2018;154(2):319-32 e3. doi:10.1053/j.gastro.2017.06.067. [PubMed: 28774845]

2. O'Shea KM, Aceves SS, Dellon ES, Gupta SK, Spergel JM, Furuta GT et al. Pathophysiology of Eosinophilic Esophagitis. Gastroenterology 2018;154(2):333-45. doi:10.1053/j.gastro.2017.06.065. [PubMed: 28757265]

3. Moawad FJ. Eosinophilic Esophagitis: Incidence and Prevalence. Gastrointest Endosc Clin N Am 2018;28(1):15-25. doi:10.1016/j.giec.2017.07.001. [PubMed: 29129296]

4. Shaheen NJ, Mukkada V, Eichinger CS, Schofield H, Todorova L, Falk GW. Natural history of eosinophilic esophagitis: a systematic review of epidemiology and disease course. Dis Esophagus 2018. doi:10.1093/dote/doy015.

5. Dellon ES, Kim HP, Sperry SL, Rybnicek DA, Woosley JT, Shaheen NJ. A phenotypic analysis shows that eosinophilic esophagitis is a progressive fibrostenotic disease. Gastrointest Endosc 2014;79(4):577-85 e4. doi:10.1016/j.gie.2013.10.027. [PubMed: 24275329]

6. Furuta GT, Katzka DA. Eosinophilic Esophagitis. N Engl J Med 2015;373(17):1640-8. doi:10.1056/ NEJMra1502863. [PubMed: 26488694]

7. Warners MJ, Oude Nijhuis RAB, de Wijkerslooth LRH, Smout A, Bredenoord AJ. The natural course of eosinophilic esophagitis and long-term consequences of undiagnosed disease in a large cohort. Am J Gastroenterol 2018;113(6):836-44. doi:10.1038/s41395-018-0052-5. [PubMed: 29700481 * This study evaluated the natural course of EoE in 721 patients that supports a progression from inflammatory to a fibrostenotic phenotype. The authors identified diagnostic delay and male gender as the major risk factors for stricture presence.

8. Menard-Katcher C, Benitez AJ, Pan Z, Ahmed FN, Wilkins BJ, Capocelli KE et al. Influence of Age and Eosinophilic Esophagitis on Esophageal Distensibility in a Pediatric Cohort. Am J Gastroenterol 2017;112(9):1466-73. doi:10.1038/ajg.2017.131. [PubMed: 28508868] ** This study showed that pathologic remodeling occurs in pediatric EoE and affects esophageal distensibility. The authors calls for the need for early diagnosis and initiating therapy prior to onset of disease complications.

9. Nhu QM, Aceves SS. Tissue Remodeling in Chronic Eosinophilic Esophageal Inflammation: Parallels in Asthma and Therapeutic Perspectives. Front Med (Lausanne) 2017;4:128. doi:10.3389/ fmed.2017.00128. [PubMed: 28831387]

10. Lucendo AJ, Arias-Gonzalez L, Molina-Infante J, Arias A. Determinant factors of quality of life in adult patients with eosinophilic esophagitis. United European Gastroenterol J 2018;6(1):38-45. doi:10.1177/2050640617707095.

11. Mukkada V, Falk GW, Eichinger CS, King D, Todorova L, Shaheen NJ. Health-Related Quality of Life and Costs Associated With Eosinophilic Esophagitis: A Systematic Review. Clin Gastroenterol Hepatol 2018;16(4):495-503 e8. doi:10.1016/j.cgh.2017.06.036. [PubMed: 28655543]

12. Aceves SS, King E, Collins MH, Yang GY, Capocelli KE, Abonia JP et al. Alignment of parentand child-reported outcomes and histology in eosinophilic esophagitis across multiple CEGIR sites. J Allergy Clin Immunol 2018;142(1):130-8 e1. doi:10.1016/j.jaci.2018.05.014. [PubMed: 29852258]

13. Lynch MK, Barnes MJ, Dimmitt RA, Martin L, Rothenberg ME, Goodin BR. Disease-Related Predictors of Health-Related Quality of Life in Youth With Eosinophilic Esophagitis. J Pediatr Psychol 2018;43(4):464-71. doi:10.1093/jpepsy/jsx128. [PubMed: 29048518]

14. Lynch MK, Dimmitt RA, Goodin BR. Evidence of Disturbed Sleep in Children With Eosinophilic Esophagitis and Persistent Epigastric Pain. J Pediatr Psychol 2018;43(3):331-41. doi:10.1093/ jpepsy/jsx117. [PubMed: 29048495] 
15. Ettyreddy AR, Sink JR, Georg MW, Kitsko DJ, Simons JP. Association between Eosinophilic Esophagitis and Esophageal Food Impaction in the Pediatric Population. Otolaryngol Head Neck Surg 2018:194599818779049. doi:10.1177/0194599818779049.

16. Sun RW, Bonilla-Velez J, Pesek RD, Johnson AB, Cleves MA, Richter GT. Eosinophilic esophagitis in children under the age of 5 years: Clinical characteristics. Laryngoscope 2018;128(4):798-805. doi:10.1002/lary.26838. [PubMed: 28865084]

17. Falk GW. Clinical presentation of eosinophilic esophagitis in adults. Gastroenterol Clin North Am 2014;43(2):231-42. doi:10.1016/j.gtc.2014.02.009. [PubMed: 24813512]

18. Paquet B, Begin P, Paradis L, Drouin E, Des Roches A. High rate of failure to thrive in a pediatric cohort with eosinophilic esophagitis. Ann Allergy Asthma Immunol 2016;116(1):73-4 e1. doi: 10.1016/j.anai.2015.09.015. [PubMed: 26507711]

19. Wolf WA, Piazza NA, Gebhart JH, Rusin S, Covey S, Higgins LL et al. Association Between Body Mass Index and Clinical and Endoscopic Features of Eosinophilic Esophagitis. Dig Dis Sci 2017;62(1):143-9. doi:10.1007/s10620-016-4357-1. [PubMed: 27778205]

20. Mehta P, Furuta GT, Brennan T, Henry ML, Maune NC, Sundaram SS et al. Nutritional State and Feeding Behaviors of Children With Eosinophilic Esophagitis and Gastroesophageal Reflux Disease. J Pediatr Gastroenterol Nutr 2018;66(4):603-8. doi:10.1097/MPG.0000000000001741. [PubMed: 28906318]

21. Reed CC, Koutlas NT, Robey BS, Hansen J, Dellon ES. Prolonged Time to Diagnosis of Eosinophilic Esophagitis Despite Increasing Knowledge of the Disease. Clin Gastroenterol Hepatol 2018. doi:10.1016/j.cgh.2018.01.028.

22. Leiman DA, Kochar B, Posner S, Fan C, Patel A, Shaheen O et al. A diagnosis of eosinophilic esophagitis is associated with increased life insurance premiums. Dis Esophagus 2018. doi: 10.1093/dote/doy008.

23. Godwin B, Liacouras C, Mehta V, Eisenberg J, Agawu A, Brown-Whitehorn T et al. A Review of Tertiary Referrals for Management of Pediatric Esophageal Eosinophilia. Front Pediatr 2018;6:173. doi:10.3389/fped.2018.00173. [PubMed: 29974044]

24. Harris BR, Hon E, Gupta SK. Implementation of guidelines in eosinophilic esophagitis at an academic pediatric practice. Dis Esophagus 2018. doi:10.1093/dote/doy029.

25. Straumann A, Katzka DA. Diagnosis and Treatment of Eosinophilic Esophagitis. Gastroenterology 2018;154(2):346-59. doi:10.1053/j.gastro.2017.05.066. [PubMed: 28756235]

26. Dellon ES, Liacouras CA, Molina-Infante J, Furuta GT, Spergel JM, Zevit N et al. Updated international consensus diagnostic criteria for eosinophilic esophagitis: Proceedings of the AGREE conference. Gastroenterology 2018. doi:10.1053/j.gastro.2018.07.009.** The AGREE conference updated the diagnostic algorithm for EoE, with removal of proton-pump inhibitor trial requirement for EoE diagnosis.

27. Hirano I, Moy N, Heckman MG, Thomas CS, Gonsalves N, Achem SR. Endoscopic assessment of the oesophageal features of eosinophilic oesophagitis: validation of a novel classification and grading system. Gut 2013;62(4):489-95. doi:10.1136/gutjnl-2011-301817. [PubMed: 22619364]

28. Dellon ES, Cotton CC, Gebhart JH, Higgins LL, Beitia R, Woosley JT et al. Accuracy of the Eosinophilic Esophagitis Endoscopic Reference Score in Diagnosis and Determining Response to Treatment. Clin Gastroenterol Hepatol 2016;14(1):31-9. doi:10.1016/j.cgh.2015.08.040. [PubMed: 26404868]

29. Wechsler JB, Bolton SM, Amsden K, Wershil BK, Hirano I, Kagalwalla AF. Eosinophilic Esophagitis Reference Score Accurately Identifies Disease Activity and Treatment Effects in Children. Clin Gastroenterol Hepatol 2018;16(7):1056-63. doi:10.1016/j.cgh.2017.12.019. [PubMed: 29248734]

30. Dellon ES, Gonsalves N, Hirano I, Furuta GT, Liacouras CA, Katzka DA et al. ACG clinical guideline: Evidenced based approach to the diagnosis and management of esophageal eosinophilia and eosinophilic esophagitis (EoE). Am J Gastroenterol 2013;108(5):679-92; quiz 93. doi: 10.1038/ajg.2013.71. [PubMed: 23567357]

31. Wang J, Park JY, Huang R, Souza RF, Spechler SJ, Cheng E. Obtaining adequate lamina propria for subepithelial fibrosis evaluation in pediatric eosinophilic esophagitis. Gastrointest Endosc 2018;87(5):1207-14 e3. doi:10.1016/j.gie.2017.12.020. [PubMed: 29309779] * This study 
reconfirmed that EoE is a patchy disease, but also identified that subepithelial fibrosis was already present in newly diagnosed EoE in children, which tended to appear in the middle or distal esophagus. The authors proposed that at least 7 middle-distal esophageal biopsy specimens were required to optimally detect EoE subepithelial fibrosis.

32. Friedlander JA, DeBoer EM, Soden JS, Furuta GT, Menard-Katcher CD, Atkins D et al. Unsedated transnasal esophagoscopy for monitoring therapy in pediatric eosinophilic esophagitis.

Gastrointest Endosc 2016;83(2):299-306 e1. doi:10.1016/j.gie.2015.05.044. [PubMed: 26142551]

* This study evaluated unsedated transnasal endoscopy (TNE) in EoE in a small cohort, but demonstrated that the procedure was feasible, preferred, and effective in monitoring the proximal esophageal mucosa of children with EoE.

33. Nelson MJ, Miller FH, Moy N, Zalewski A, Gonsalves N, Gregory DL et al. Comparison of endoscopy and radiographic imaging for detection of esophageal inflammation and remodeling in adults with eosinophilic esophagitis. Gastrointest Endosc 2018;87(4):962-8. doi:10.1016/j.gie. 2017.09.037. [PubMed: 28989006]

34. Hirano I, Pandolfino JE, Boeckxstaens GE. Functional Lumen Imaging Probe for the Management of Esophageal Disorders: Expert Review From the Clinical Practice Updates Committee of the AGA Institute. Clin Gastroenterol Hepatol 2017;15(3):325-34. doi:10.1016/j.cgh.2016.10.022. [PubMed: 28212976]

35. Ahuja NK, Clarke JO. The Role of Impedance Planimetry in the Evaluation of Esophageal Disorders. Curr Gastroenterol Rep 2017;19(2):7. doi:10.1007/s11894-017-0544-2. [PubMed: 28220362]

36. Nicodeme F, Hirano I, Chen J, Robinson K, Lin Z, Xiao Y et al. Esophageal distensibility as a measure of disease severity in patients with eosinophilic esophagitis. Clin Gastroenterol Hepatol 2013;11(9):1101-7 e1. doi:10.1016/j.cgh.2013.03.020. [PubMed: 23591279] * This study demonstrated in EoE patients that reduced esophageal distensibility could be used to predict food impaction risk and the need for esophageal dilation.

37. Lin Z, Kahrilas PJ, Xiao Y, Nicodeme F, Gonsalves N, Hirano I et al. Functional luminal imaging probe topography: an improved method for characterizing esophageal distensibility in eosinophilic esophagitis. Therap Adv Gastroenterol 2013;6(2):97-107. doi:10.1177/1756283X12470017.

38. Carlson DA, Hirano I, Zalewski A, Gonsalves N, Lin Z, Pandolfino JE. Improvement in Esophageal Distensibility in Response to Medical and Diet Therapy in Eosinophilic Esophagitis. Clin Transl Gastroenterol 2017;8(10):e119. doi:10.1038/ctg.2017.47. [PubMed: 28981080]

39. Katzka DA, Smyrk TC, Alexander JA, Geno DM, Beitia RA, Chang AO et al. Accuracy and Safety of the Cytosponge for Assessing Histologic Activity in Eosinophilic Esophagitis: A Two-Center Study. Am J Gastroenterol 2017;112(10):1538-44. doi:10.1038/ajg.2017.244. [PubMed: 28809387] ** This prospective two-center cross-sectional study showed that the minimally invasive cytosponge was safe, well tolerated, preferred by patients, and accurate, with the sensitivity and specificity of the cytosponge to assess EoE histologic activity being $75 \%$ and $86 \%$, respectively.

40. Katzka DA, Geno DM, Ravi A, Smyrk TC, Lao-Sirieix P, Miremadi A et al. Accuracy, safety, and tolerability of tissue collection by Cytosponge vs endoscopy for evaluation of eosinophilic esophagitis. Clin Gastroenterol Hepatol 2015;13(1):77-83 e2. doi:10.1016/j.cgh.2014.06.026. [PubMed: 24997328]

41. Furuta GT, Kagalwalla AF, Lee JJ, Alumkal P, Maybruck BT, Fillon S et al. The oesophageal string test: a novel, minimally invasive method measures mucosal inflammation in eosinophilic oesophagitis. Gut 2013;62(10):1395-405. doi:10.1136/gutjnl-2012-303171. [PubMed: 22895393]

** This study demonstrated the novel, minimally invasive esophageal string test that measured eosinophil-derived proteins in luminal secretions to assess esophageal eosinophilic inflammation in pediatric EoE.

42. Kern E, Lin D, Larson A, Yang GY, Taft T, Zalewski A et al. Prospective assessment of the diagnostic utility of esophageal brushings in adults with eosinophilic esophagitis. Dis Esophagus 2016;29(1):48-53. doi:10.1111/dote.12304. [PubMed: 25515533]

43. Smadi Y, Deb C, Bornstein J, Safder S, Horvath K, Mehta D. Blind esophageal brushing offers a safe and accurate method to monitor inflammation in children and young adults with eosinophilic esophagitis. Dis Esophagus 2018. doi:10.1093/dote/doy056. 
44. Kephart GM, Alexander JA, Arora AS, Romero Y, Smyrk TC, Talley NJ et al. Marked deposition of eosinophil-derived neurotoxin in adult patients with eosinophilic esophagitis. Am J Gastroenterol 2010;105(2):298-307. doi:10.1038/ajg.2009.584. [PubMed: 19888203]

45. Barrett C, Choksi Y, Vaezi MF. Mucosal Impedance: a New Approach to Diagnosing Gastroesophageal Reflux Disease and Eosinophilic Esophagitis. Curr Gastroenterol Rep 2018;20(7):33. doi:10.1007/s11894-018-0639-4. [PubMed: 29886566]

46. Patel DA, Vaezi MF. Utility of esophageal mucosal impedance as a diagnostic test for esophageal disease. Curr Opin Gastroenterol 2017;33(4):277-84. doi:10.1097/MOG.0000000000000367. [PubMed: 28437259]

47. Choksi Y, Lal P, Slaughter JC, Sharda R, Parnell J, Higginbotham T et al. Esophageal Mucosal Impedance Patterns Discriminate Patients With Eosinophilic Esophagitis From Patients With GERD. Clin Gastroenterol Hepatol 2018;16(5):664-71 e1. doi:10.1016/j.cgh.2017.12.020. [PubMed: 29248733] * This study evaluated mucosal impedance measurements at 2, 5, and $10 \mathrm{~cm}$ from the squamocolumnar junction and demonstrated that EoE patients had a unique mucosal impedance pattern of low values along the esophageal axis.

48. Ates F, Yuksel ES, Higginbotham T, Slaughter JC, Mabary J, Kavitt RT et al. Mucosal impedance discriminates GERD from non-GERD conditions. Gastroenterology 2015;148(2):334-43. doi: 10.1053/j.gastro.2014.10.010. [PubMed: 25448923]

49. Lowry MA, Vaezi MF, Correa H, Higginbotham T, Slaughter JC, Acra S. Mucosal Impedance Measurements Differentiate Pediatric Patients With Active Versus Inactive Eosinophilic Esophagitis. J Pediatr Gastroenterol Nutr 2018;67(2):198-203. doi:10.1097/MPG. 0000000000001943. [PubMed: 29543695] ** This study demonstrated that mucosal impedance was lower in active EoE at 2, 5, and $10 \mathrm{~cm}$ above the squamocolumnar junction, compared to inactive EoE, non-erosive reflux disease, and controls. The study supports that mucosal impedance is a promising rapid and less-invasive method to monitor EoE activity.

50. Ishihara S, Shoda T, Ishimura N, Ohta S, Ono J, Azuma Y et al. Serum Biomarkers for the Diagnosis of Eosinophilic Esophagitis and Eosinophilic Gastroenteritis. Intern Med 2017;56(21): 2819-25. doi:10.2169/internalmedicine.8763-16. [PubMed: 28943560]

51. Dellon ES, Rusin S, Gebhart JH, Covey S, Higgins LL, Beitia R et al. Utility of a Noninvasive Serum Biomarker Panel for Diagnosis and Monitoring of Eosinophilic Esophagitis: A Prospective Study. Am J Gastroenterol 2015;110(6):821-7. doi:10.1038/ajg.2015.57. [PubMed: 25781367]

52. Dellon ES, Higgins LL, Beitia R, Rusin S, Woosley JT, Veerappan R et al. Prospective assessment of serum periostin as a biomarker for diagnosis and monitoring of eosinophilic oesophagitis. Aliment Pharmacol Ther 2016;44(2):189-97. doi:10.1111/apt.13672. [PubMed: 27193351]

53. Wright BL, Ochkur SI, Olson NS, Shim KP, Jacobsen EA, Rank MA et al. Normalized serum eosinophil peroxidase levels are inversely correlated with esophageal eosinophilia in eosinophilic esophagitis. Dis Esophagus 2018;31(2). doi:10.1093/dote/dox139.

54. Min SB, Nylund CM, Baker TP, Ally M, Reinhardt B, Chen YJ et al. Longitudinal Evaluation of Noninvasive Biomarkers for Eosinophilic Esophagitis. J Clin Gastroenterol 2017;51(2):127-35. doi:10.1097/MCG.0000000000000621. [PubMed: 27479142]

55. Hines BT, Rank MA, Wright BL, Marks LA, Hagan JB, Straumann A et al. Minimally-invasive biomarker studies in eosinophilic esophagitis: a systematic review. Ann Allergy Asthma Immunol 2018. doi:10.1016/j.anai.2018.05.005.

56. Erwin EA, Rhoda DA, Redmond M, Ly JB, Russo JM, Hill ID et al. Using Serum IgE Antibodies to Predict Esophageal Eosinophilia in Children. J Pediatr Gastroenterol Nutr 2017;65(5):520-5. doi:10.1097/MPG.0000000000001553. [PubMed: 28981449]

57. Clayton F, Fang JC, Gleich GJ, Lucendo AJ, Olalla JM, Vinson LA et al. Eosinophilic esophagitis in adults is associated with IgG4 and not mediated by IgE. Gastroenterology 2014;147(3):602-9. doi:10.1053/j.gastro.2014.05.036. [PubMed: 24907494]

58. Mohammad N, Avinashi V, Chan E, Vallance BA, Portales-Casamar E, Bush JW. Pediatric Eosinophilic Esophagitis Is Associated With Increased Lamina Propria Immunoglobulin G4Positive Plasma Cells. J Pediatr Gastroenterol Nutr 2018. doi:10.1097/MPG.0000000000001949.

59. Rosenberg CE, Mingler MK, Caldwell JM, Collins MH, Fulkerson PC, Morris DW et al. Esophageal IgG4 levels correlate with histopathologic and transcriptomic features in eosinophilic esophagitis. Allergy 2018. doi:10.1111/all.13486. 
60. Schuyler AJ, Wilson JM, Tripathi A, Commins SP, Ogbogu PU, Kruzsewski PG et al. Specific IgG4 antibodies to cow's milk proteins in pediatric patients with eosinophilic esophagitis. J Allergy Clin Immunol 2018;142(1):139-48 e12. doi:10.1016/j.jaci.2018.02.049. [PubMed: 29678750]

61. Dellon ES, Lin L, Beitia R, Moran TP, Qian Y. Serum autoantibodies against epithelial cell adhesion molecules as disease biomarkers of eosinophilic esophagitis. Clin Exp Allergy 2018;48(3):343-6. doi:10.1111/cea.13070. [PubMed: 29193433]

62. Nhu QM, Aceves SS. Medical and dietary management of eosinophilic esophagitis. Ann Allergy Asthma Immunol 2018;121(2):156-61. doi:10.1016/j.anai.2018.05.006. [PubMed: 29753831]

63. Sastre J, Davila I. Dupilumab: A New Paradigm for the Treatment of Allergic Diseases. J Investig Allergol Clin Immunol 2018;28(3):139-50. doi:10.18176/jiaci.0254.

64. Lipka S, Keshishian J, Boyce HW, Estores D, Richter JE. The natural history of steroid-naive eosinophilic esophagitis in adults treated with endoscopic dilation and proton pump inhibitor therapy over a mean duration of nearly 14 years. Gastrointest Endosc 2014;80(4):592-8. doi: 10.1016/j.gie.2014.02.012. [PubMed: 24703087]

65. Runge TM, Eluri S, Woosley JT, Shaheen NJ, Dellon ES. Control of inflammation decreases the need for subsequent esophageal dilation in patients with eosinophilic esophagitis. Dis Esophagus 2017;30(7):1-7. doi:10.1093/dote/dox042.

66. Dellon ES, Sheikh A, Speck O, Woodward K, Whitlow AB, Hores JM et al. Viscous topical is more effective than nebulized steroid therapy for patients with eosinophilic esophagitis. Gastroenterology 2012;143(2):321-4 e1. doi:10.1053/j.gastro.2012.04.049. [PubMed: 22561055]

67. Gupta SK, Vitanza JM, Collins MH. Efficacy and safety of oral budesonide suspension in pediatric patients with eosinophilic esophagitis. Clin Gastroenterol Hepatol 2015;13(1):66-76 e3. doi: 10.1016/j.cgh.2014.05.021. [PubMed: 24907502]

68. Miehlke S, Hruz P, Vieth M, Bussmann C, von Arnim U, Bajbouj M et al. A randomised, doubleblind trial comparing budesonide formulations and dosages for short-term treatment of eosinophilic oesophagitis. Gut 2016;65(3):390-9. doi:10.1136/gutjnl-2014-308815. [PubMed: 25792708 ** This study evaluated two different budesonide formulations, effervescent tablet for orodispersible use and viscous suspension, and demonstrated that both were highly effective and safe for short-term treatment of EoE, with patients preferring the effervescent tablet.

69. Dohil R, Newbury R, Fox L, Bastian J, Aceves S. Oral viscous budesonide is effective in children with eosinophilic esophagitis in a randomized, placebo-controlled trial. Gastroenterology 2010;139(2):418-29. doi:10.1053/j.gastro.2010.05.001. [PubMed: 20457157]

70. Butz BK, Wen T, Gleich GJ, Furuta GT, Spergel J, King E et al. Efficacy, dose reduction, and resistance to high-dose fluticasone in patients with eosinophilic esophagitis. Gastroenterology 2014;147(2):324-33 e5. doi:10.1053/j.gastro.2014.04.019. [PubMed: 24768678] ** This study demonstrated that high-dose fluticasone induced histologic remission after 3 months of treatment and that responders could have the dose reduced by $50 \%$ in $73 \%$ to $93 \%$ of the responding patients.

71. Straumann A, Miehlke S, Vieth M, Schlag C, von Arnim U, Molina-Infante J et al. Sa1145 Predictors for Early Versus Delayed Response to Budesonide Orodispersible Tablets in Eosinophilic Esophagitis Treatment: A Subgroup Analysis from the Pivotal Trial Eos-1. Gastroenterology 2018;154(6):S-256-S-7. doi:10.1016/S0016-5085(18)31226-5.

72. Blanchard C, Mingler MK, Vicario M, Abonia JP, Wu YY, Lu TX et al. IL-13 involvement in eosinophilic esophagitis: transcriptome analysis and reversibility with glucocorticoids. J Allergy Clin Immunol 2007;120(6):1292-300. doi:10.1016/j.jaci.2007.10.024. [PubMed: 18073124]

73. Katzka DA, Tadi R, Smyrk TC, Katarya E, Sharma A, Geno DM et al. Effects of topical steroids on tight junction proteins and spongiosis in esophageal epithelia of patients with eosinophilic esophagitis. Clin Gastroenterol Hepatol 2014;12(11):1824-9 e1. doi:10.1016/j.cgh.2014.02.039. [PubMed: 24681080]

74. Simon D, Page B, Vogel M, Bussmann C, Blanchard C, Straumann A et al. Evidence of an abnormal epithelial barrier in active, untreated and corticosteroid-treated eosinophilic esophagitis. Allergy 2018;73(1):239-47. doi:10.1111/all.13244. [PubMed: 28712126]

75. Andreae DA, Hanna MG, Magid MS, Malerba S, Andreae MH, Bagiella E et al. Swallowed Fluticasone Propionate Is an Effective Long-Term Maintenance Therapy for Children With 
Eosinophilic Esophagitis. Am J Gastroenterol 2016;111(8):1187-97. doi:10.1038/ajg.2016.238. [PubMed: 27325220] ** This study demonstrated in pediatric EoE that long-term maintenance dosing of aerosolized and swallowed fluticasone was safe and that it improved histologic inflammation, lamina propria fibrosis, endoscopic features and clinical symptoms.

76. Fable JM, Fernandez M, Goodine S, Lerer T, Sayej WN. Retrospective Comparison of Fluticasone Propionate and Oral Viscous Budesonide in Children With Eosinophilic Esophagitis. J Pediatr Gastroenterol Nutr 2018;66(1):26-32. doi:10.1097/MPG.0000000000001626. [PubMed: 28489670]

77. Reed CC, Fan C, Koutlas N, Stefanadis Z, Eluri S, Shaheen NJ et al. Compounded Oral Viscous Budesonide is Effective and Provides a Durable Response in Eosinophilic Esophagitis. HSOA J Gastroenterol Hepatol Res 2018;7(1):2509-15. [PubMed: 29608193]

78. Greuter T, Bussmann C, Safroneeva E, Schoepfer AM, Biedermann L, Vavricka SR et al. LongTerm Treatment of Eosinophilic Esophagitis With Swallowed Topical Corticosteroids: Development and Evaluation of a Therapeutic Concept. Am J Gastroenterol 2017;112(10):152735. doi:10.1038/ajg.2017.202. [PubMed: 28719593] ** This study proposed the concept of deep remission as a therapeutic goal for EoE and also proposed and evaluated an induction-maintenance corticosteroid protocol for EoE.

79. Rubinstein E, Hait EE, Mitchell PD, Lee JJ. Every-other-day Dosing of Oral Viscous Budesonide Is not Effective in the Management of Eosinophlic Esophagitis. J Pediatr Gastroenterol Nutr 2018;66(3):395-7. doi:10.1097/MPG.0000000000001718. [PubMed: 28837508]

80. Lindberg GM, Van Eldik R, Saboorian MH. A case of herpes esophagitis after fluticasone propionate for eosinophilic esophagitis. Nat Clin Pract Gastroenterol Hepatol 2008;5(9):527-30. doi:10.1038/ncpgasthep1225. [PubMed: 18758471]

81. Alexander JA. Steroid treatment of eosinophilic esophagitis in adults. Gastroenterol Clin North Am 2014;43(2):357-73. doi:10.1016/j.gtc.2014.02.001. [PubMed: 24813521]

82. Hsu S, Wood C, Pan Z, Rahat H, Zeitler P, Fleischer D et al. Adrenal Insufficiency in Pediatric Eosinophilic Esophagitis Patients Treated with Swallowed Topical Steroids. Pediatr Allergy Immunol Pulmonol 2017;30(3):135-40. doi:10.1089/ped.2017.0779. [PubMed: 29062584]

83. Philpott H, Dougherty MK, Reed CC, Caldwell M, Kirk D, Torpy DJ et al. Systematic review: adrenal insufficiency secondary to swallowed topical corticosteroids in eosinophilic oesophagitis. Aliment Pharmacol Ther 2018;47(8):1071-8. doi:10.1111/apt.14573. [PubMed: 29508432]

84. Dellon ES, Speck O, Woodward K, Gebhart JH, Madanick RD, Levinson S et al. Clinical and endoscopic characteristics do not reliably differentiate PPI-responsive esophageal eosinophilia and eosinophilic esophagitis in patients undergoing upper endoscopy: a prospective cohort study. Am J Gastroenterol 2013;108(12):1854-60. doi:10.1038/ajg.2013.363. [PubMed: 24145677]

85. Molina-Infante J, Lucendo AJ. Proton Pump Inhibitor Therapy for Eosinophilic Esophagitis: A Paradigm Shift. Am J Gastroenterol 2017;112(12):1770-3. doi:10.1038/ajg.2017.404. [PubMed: 29087399]

86. Shoda T, Matsuda A, Nomura I, Okada N, Orihara K, Mikami H et al. Eosinophilic esophagitis versus proton pump inhibitor-responsive esophageal eosinophilia: Transcriptome analysis. J Allergy Clin Immunol 2017;139(6):2010-3 e4. doi:10.1016/j.jaci.2016.11.028. [PubMed: 28063872]

87. Molina-Infante J, Bredenoord AJ, Cheng E, Dellon ES, Furuta GT, Gupta SK et al. Proton pump inhibitor-responsive oesophageal eosinophilia: an entity challenging current diagnostic criteria for eosinophilic oesophagitis. Gut 2016;65(3):524-31. doi:10.1136/gutjnl-2015-310991. [PubMed: 26685124]

88. Moawad FJ, Schoepfer AM, Safroneeva E, Ally MR, Chen YJ, Maydonovitch CL et al. Eosinophilic oesophagitis and proton pump inhibitor-responsive oesophageal eosinophilia have similar clinical, endoscopic and histological findings. Aliment Pharmacol Ther 2014;39(6):603-8. doi:10.1111/apt.12636. [PubMed: 24461332]

89. Zhang X, Cheng E, Huo X, Yu C, Zhang Q, Pham TH et al. Omeprazole blocks STAT6 binding to the eotaxin-3 promoter in eosinophilic esophagitis cells. PLoS One 2012;7(11):e50037. doi: 10.1371/journal.pone.0050037. [PubMed: 23185525]

90. Cheng E, Zhang X, Huo X, Yu C, Zhang Q, Wang DH et al. Omeprazole blocks eotaxin-3 expression by oesophageal squamous cells from patients with eosinophilic oesophagitis and 
GORD. Gut 2013;62(6):824-32. doi:10.1136/gutjnl-2012-302250. [PubMed: 22580413] * This study demonstrated that omeprazole inhibited IL-4/IL-13-induced eotaxin-3 expression in esophageal squamous epithelial cells, suggesting that PPI therapy has therapeutic potential beyond acid suppression.

91. Lucendo AJ, Arias A, Molina-Infante J. Efficacy of Proton Pump Inhibitor Drugs for Inducing Clinical and Histologic Remission in Patients With Symptomatic Esophageal Eosinophilia: A Systematic Review and Meta-Analysis. Clin Gastroenterol Hepatol 2016;14(1):13-22 e1. doi: 10.1016/j.cgh.2015.07.041. [PubMed: 26247167] * This meta-analysis of 33 studies of 188 children and 431 adult patients with symptomatic esophageal eosinophilia showed that PPI therapy induced clinical and histologic remission in $60.8 \%$ and $50.5 \%$ of the patients, respectively.

92. Molina-Infante J, Prados-Manzano R, Gonzalez-Cordero PL. The role of proton pump inhibitor therapy in the management of eosinophilic esophagitis. Expert Rev Clin Immunol 2016;12(9): 945-52. doi:10.1080/1744666X.2016.1178574. [PubMed: 27097787]

93. Gutierrez-Junquera C, Fernandez-Fernandez S, Cilleruelo ML, Rayo A, Echeverria L, Borrell B et al. Long-term Treatment With Proton Pump Inhibitors Is Effective in Children With Eosinophilic Esophagitis. J Pediatr Gastroenterol Nutr 2018. doi:10.1097/MPG.0000000000001952.

94. Gonsalves N, Yang GY, Doerfler B, Ritz S, Ditto AM, Hirano I. Elimination diet effectively treats eosinophilic esophagitis in adults; food reintroduction identifies causative factors. Gastroenterology 2012;142(7):1451-9 e1; quiz e14-5. doi:10.1053/j.gastro.2012.03.001. [PubMed: 22391333]

95. Lieberman JA, Morotti RA, Konstantinou GN, Yershov O, Chehade M. Dietary therapy can reverse esophageal subepithelial fibrosis in patients with eosinophilic esophagitis: a historical cohort. Allergy 2012;67(10):1299-307. doi:10.1111/j.1398-9995.2012.02881.x. [PubMed: 22913672]

96. Arias A, Gonzalez-Cervera J, Tenias JM, Lucendo AJ. Efficacy of dietary interventions for inducing histologic remission in patients with eosinophilic esophagitis: a systematic review and meta-analysis. Gastroenterology 2014;146(7):1639-48. doi:10.1053/j.gastro.2014.02.006. [PubMed: 24534634]

97. Warners MJ, Vlieg-Boerstra BJ, Verheij J, van Hamersveld PHP, van Rhijn BD, Van Ampting MTJ et al. Esophageal and Small Intestinal Mucosal Integrity in Eosinophilic Esophagitis and Response to an Elemental Diet. Am J Gastroenterol 2017;112(7):1061-71. doi:10.1038/ajg.2017.107. [PubMed: 28417991]

98. Warners MJ, Vlieg-Boerstra BJ, Verheij J, van Rhijn BD, Van Ampting MT, Harthoorn LF et al. Elemental diet decreases inflammation and improves symptoms in adult eosinophilic oesophagitis patients. Aliment Pharmacol Ther 2017;45(6):777-87. doi:10.1111/apt.13953. [PubMed: 28112427]

99. Kagalwalla AF, Wechsler JB, Amsden K, Schwartz S, Makhija M, Olive A et al. Efficacy of a 4Food Elimination Diet for Children With Eosinophilic Esophagitis. Clin Gastroenterol Hepatol 2017;15(11):1698-707 e7. doi:10.1016/j.cgh.2017.05.048. [PubMed: 28603055]

100. Kagalwalla AF, Sentongo TA, Ritz S, Hess T, Nelson SP, Emerick KM et al. Effect of six-food elimination diet on clinical and histologic outcomes in eosinophilic esophagitis. Clin Gastroenterol Hepatol 2006;4(9):1097-102. doi:10.1016/j.cgh.2006.05.026. [PubMed: 16860614]

101. Molina-Infante J, Martin-Noguerol E, Alvarado-Arenas M, Porcel-Carreno SL, Jimenez-Timon S, Hernandez-Arbeiza FJ. Selective elimination diet based on skin testing has suboptimal efficacy for adult eosinophilic esophagitis. J Allergy Clin Immunol 2012;130(5):1200-2. doi:10.1016/ j.jaci.2012.06.027. [PubMed: 22867695]

102. Eckmann JD, Ravi K, Katzka DA, Davis DR, See JA, Geno DR et al. Efficacy of Atopy Patch Testing in Directed Dietary Therapy of Eosinophilic Esophagitis: A Pilot Study. Dig Dis Sci 2018;63(3):694-702. doi:10.1007/s10620-018-4928-4. [PubMed: 29349695]

103. Warners MJ, Terreehorst I, van den Wijngaard RM, Akkerdaas J, van Esch B, van Ree R et al. Abnormal Responses to Local Esophageal Food Allergen Injections in Adult Patients With Eosinophilic Esophagitis. Gastroenterology 2018;154(1):57-60 e2. doi:10.1053/j.gastro. 2017.08.062. [PubMed: 28870530] ** The novel study evaluated esophageal mucosal food allergen injection in a small cohort and showed induction of acute and/or delayed responses in EoE patients but not in non-EoE controls. 
104. Molina-Infante J, Arias A, Alcedo J, Garcia-Romero R, Casabona-Frances S, Prieto-Garcia A et al. Step-up empiric elimination diet for pediatric and adult eosinophilic esophagitis: The 2-4-6 study. J Allergy Clin Immunol 2017. doi:10.1016/j.jaci.2017.08.038.** This study proposed and evaluated the 2-4-6 step up elimination diet therapy protocol for EoE which was effective and identified food triggers of EoE early, resulting in less unnecessary dietary restrictions, less number of endoscopic evaluations, and a shorter diagnostic process.

105. Philpott H, Dellon E. Histologic improvement after 6 weeks of dietary elimination for eosinophilic esophagitis may be insufficient to determine efficacy. Asia Pac Allergy 2018;8(2):e20. doi:10.5415/apallergy.2018.8.e20. [PubMed: 29732296]

106. Richter JE. Esophageal dilation in eosinophilic esophagitis. Best Pract Res Clin Gastroenterol 2015;29(5):815-28. doi:10.1016/j.bpg.2015.06.015. [PubMed: 26552780]

107. Lipka S, Kumar A, Richter JE. Successful Esophageal Dilation of Eosinophilic Esophagitis (EoE) Patients With a Previous Postdilation Complication: Start Low and Go Slow. J Clin Gastroenterol 2017. doi:10.1097/MCG.0000000000000873.

108. Runge TM, Eluri S, Cotton CC, Burk CM, Woosley JT, Shaheen NJ et al. Outcomes of Esophageal Dilation in Eosinophilic Esophagitis: Safety, Efficacy, and Persistence of the Fibrostenotic Phenotype. Am J Gastroenterol 2016;111(2):206-13. doi:10.1038/ajg.2015.399. [PubMed: 26753894]

109. Lucendo AJ, Molina-Infante J. Esophageal dilation in eosinophilic esophagitis: risks, benefits, and when to do it. Curr Opin Gastroenterol 2018;34(4):226-32. doi:10.1097/MOG. 0000000000000442. [PubMed: 29846259]

110. Menard-Katcher C, Furuta GT, Kramer RE. Dilation of Pediatric Eosinophilic Esophagitis: Adverse Events and Short-term Outcomes. J Pediatr Gastroenterol Nutr 2017;64(5):701-6. doi: 10.1097/MPG.0000000000001336. [PubMed: 28055990]

111. Moawad FJ, Molina-Infante J, Lucendo AJ, Cantrell SE, Tmanova L, Douglas KM. Systematic review with meta-analysis: endoscopic dilation is highly effective and safe in children and adults with eosinophilic oesophagitis. Aliment Pharmacol Ther 2017;46(2):96-105. doi:10.1111/apt. 14123. [PubMed: 28513085] 